\title{
Fast Enthalpy-Sensing Microsystem Operating in Continuous Flow ${ }^{+}$
}

\author{
Taoufik Mhammedi 1, ${ }^{1 *}$, Lionel Camberlein ${ }^{1}$, Frédéric Polet ${ }^{1}$, Bruno Bêche ${ }^{2,3}$ and Etienne Gaviot ${ }^{1}$ \\ 1 Laboratoire d'Acoustique de l'Université du Mans, LAUM, UMR CNRS 6613, 72085 Le Mans, France; \\ lionel.camberlein@univ-lemans.fr (L.C.); frederic.polet@univ-lemans.fr (F.P.); \\ etienne.gaviot@univ-lemans.fr (E.G.) \\ 2 Institut de Physique de Rennes, IPR, UMR CNRS 6251, 35042 Rennes, France; bruno.beche@univ-rennes1.fr \\ 3 Institut Universitaire de France (IUF), 103 Boulevard Saint-Michel, 75005 Paris, France \\ * Correspondence: Taoufik.Mhammedi@univ-lemans.fr \\ † Presented at the Eurosensors 2018 Conference, Graz, Austria, 9-12 September 2018.
}

Published: 28 November 2018

\begin{abstract}
A new microsystem, designed to detect and measure in real time the enthalpy of mixing of two fluid-constituents is presented. A preliminary approach to arrange miniaturized batch-cells allowing detecting enthalpy of dilution or mixing is first discussed. Then, a coherent rationale leading to structure devices operating in real time is formulated, considering the straightforward assessment of heat flux transducers (HFTs) capability. Basic thermodynamic observations regarding analogy between thermal and electrical systems are highlighted prior consideration of practical examples involving mixing of water and alcohols. Fundamentals about HFTs design are highlighted before presenting an adequate way to integrate both functions of mixing and measuring the entailed heat exchange as two continuously flowing fluids interact with each other. Then, a prototype of such a dedicated device is discussed with its relevant expected performance.
\end{abstract}

Keywords: heat flux transducer; planar thermopile; enthalpy of mixing; conjugated variables

\section{Introduction}

As regard industrial processes, assessing the heat exchanges occurring as two fluid constituents are mixed together is a most common requirement, especially with miniaturized systems. Being a state variable and the intensive quantity associated with thermal energy, the absolute temperature is classically the only prominent quantity to be assessed by way of calorimetry. The extensive quantity conjugated with temperature is the entropy current that should be of most valuable interest insofar as dedicated sensors would be available [1]. However, on a metrological standpoint, when small changes of temperature are involved, heat flow meters (HFMs) may supply quite relevant intelligence especially as regards detection of weak thermal changes [2]. For instance, considering phase transitions with quasi-isothermal plateaus, the HFM approach have already proved substantial metrological advantages [3,4]. In this paper we highlight a valuable way to take advantage of the planar thermopile constitutive of a generic HFM (Figure 1) so as to design a sensitive area fitted for measuring weak thermal exchanges otherwise hardly detectable in terms of ratio signal/noise. Then we describe a prototype (low impedance self-generating sensor) featuring a specific thermopile overlaid with micro-SU-8 channels [5] arranged on a glass substrate (Figures 2 and 3). As examples of specifications, processes of mixing water + ethanol and water + propan-2-ol are considered [6,7]. 


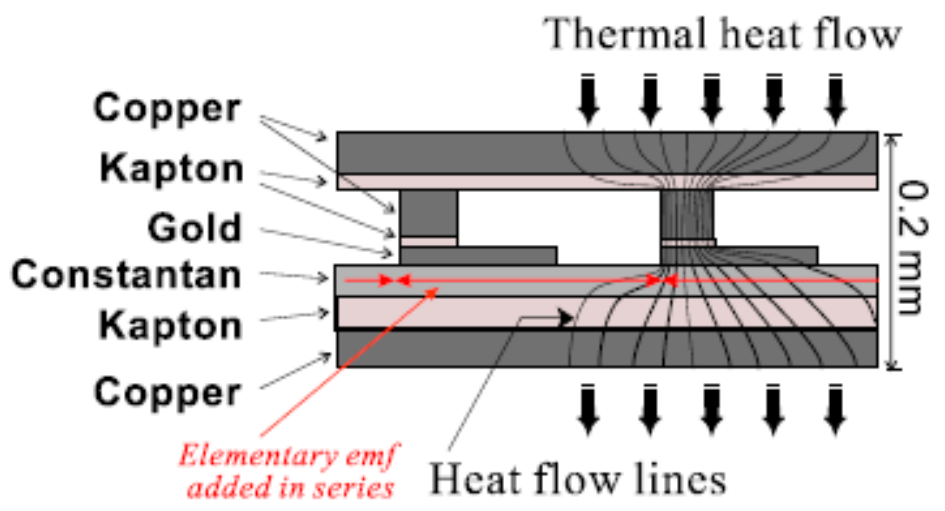

(a)

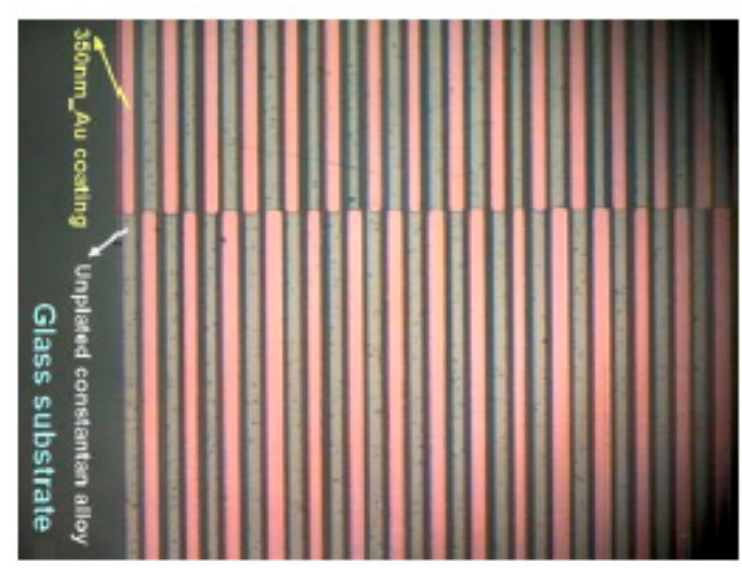

(b)

Figure 1. HFM fundamentals: (a) Cross section view of a generic structure; (b) Arrangement of a specific golden plated planar thermopile deposited on a glass substrate.

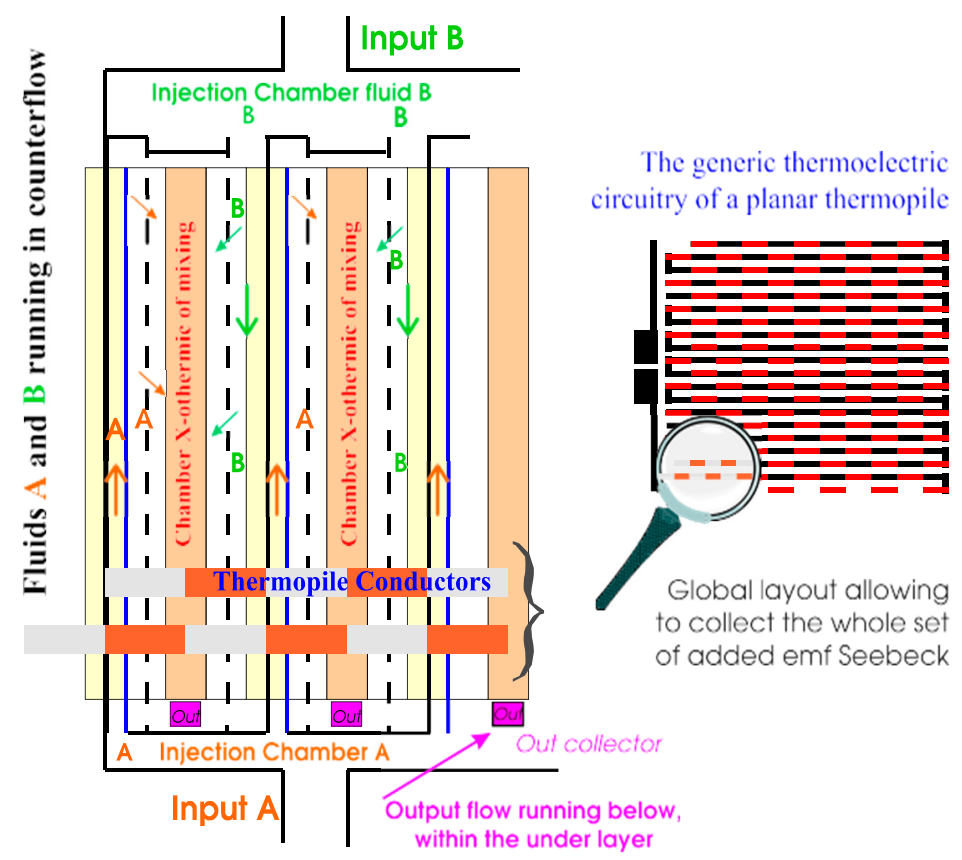

Figure 2. Main principles for constitutive parts of the self-generating microsystem (Zout $<2 \mathrm{k} \Omega$ ). 


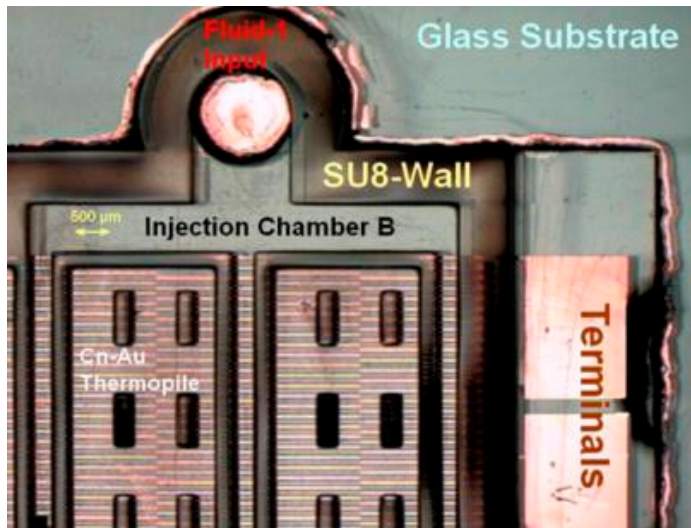

(a)

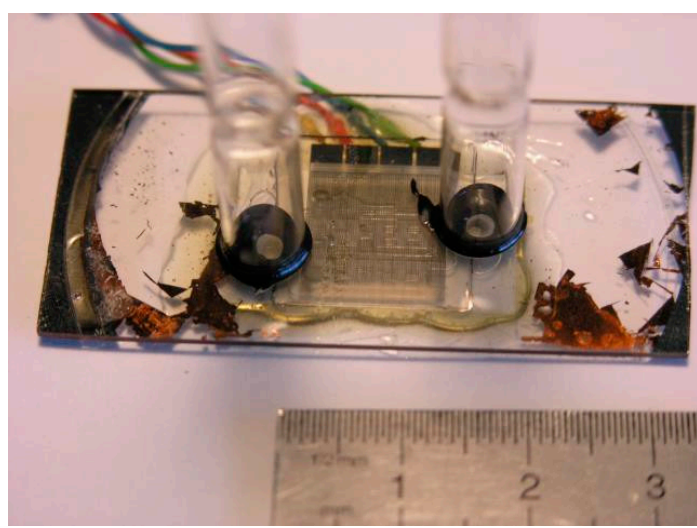

(b)

Figure 3. (a) Layout of the prototype highlighting: the glass substrate $(\lambda \mathrm{th}=1 \mathrm{~W} / \mathrm{mK})$, the AuConstantan plated planar thermopile $(\mathrm{N}=360)$, the SU8 walls $400 \mu \mathrm{m}$ in height allowing to drive and mix both fluid-constituents, and the electrical terminals; (b) Global view of the device with both fluids inputs: the output being on the other side is here invisible.

\section{Materials and Methods}

Considering calibrating, specifications for designing effective devices rely on data available in the literature, especially as regard fluid constituents to be mixed. As a mandatory requisite the constituents experimented with should be devoid of any kind of interaction with the materials constituting the systems. Prototypes involving Depron $(\lambda=0.027 \mathrm{~W} / \mathrm{mK})$, Polylactic acid (PLA: $\lambda=$ $0.13 \mathrm{~W} / \mathrm{mK})$, Polyethylene terephthalate (PET: $\lambda=0.14 \mathrm{~W} / \mathrm{mK})$, SU8 $(\lambda=0.15 \mathrm{~W} / \mathrm{mK})$, Glass $(\lambda=1$ $\mathrm{W} / \mathrm{mK})$, proved rugged enough with mixings of water with alcohols. However, PLA was advantageously replaced with PET, both materials being easily shaped with a 3D printer.

\subsection{Prerequisite for Calibration: Suitable Fluids and Ways to Handle Their Mixing}

Both Ethanol and Isopropyl-alcohol (IPA) mixed with water have been investigated, considering $[6,7]$. Products used in this paper were all "for analysis" quality products provided by Prolabo and mixed with deionised water $(10.6 \mathrm{M} \Omega . \mathrm{cm})$. Taking advantage of the Peeters \& Huyskens polynomial model, the excess enthalpies of mixing for any case of mixing may be calculated with regard to the following fitting equation where $X_{W, A}$ denote respective mole fraction of water and alcohol [with $X_{W}$ $\left.=\left(1-\mathrm{X}_{\mathrm{A}}\right)\right]$ :

$$
\Delta \mathrm{H}_{\text {mix }}=\mathrm{C}_{6 / 1} \cdot \mathrm{X}_{\mathrm{W}}^{6} \cdot \mathrm{X}_{\mathrm{A}}+\mathrm{C} 1 / 1 \cdot \mathrm{X}_{\mathrm{W}} \cdot \mathrm{X}_{\mathrm{A}}+\mathrm{C}_{1 / 2} \cdot \mathrm{X}_{\mathrm{W}} \cdot \mathrm{X}_{\mathrm{A}}^{2}
$$

Considering respective masses of water $M$ added with alcohol $m$ and the entailed ratio $r=m / M$, the mole fraction of alcohol may be written as:

$$
\mathrm{X}_{\mathrm{A}}=\frac{\mathrm{r} \cdot \mathrm{w}}{1+\mathrm{r} \cdot \mathrm{w}^{\prime}} \text { with } \mathrm{w}=\frac{\text { MWwater }}{\text { MWalc }} \text { the ratio of molecular weights. }
$$

Then, with regard to specific heat to energy balance, either the effective exchanged heat $\Delta \mathrm{Q}(\mathrm{J})$ or its related steady state heat flow $\Phi(\mathrm{W})$ may be given with:

$$
\begin{gathered}
\Delta \mathrm{Q}=\Delta \mathrm{H}_{\text {mix }} \cdot\left[\frac{\mathrm{m}}{M W_{\text {alc }}}+\frac{\mathrm{M}}{M W_{\text {water }}}\right] \\
\Phi=\frac{\mathrm{dQ}}{\mathrm{dt}}=\Delta \mathrm{H}_{\text {mix }} \cdot\left[\frac{\rho_{\text {alc }} \cdot\left(\partial V_{\text {alc }} / \partial t\right)}{M W_{\text {alco }}}+\frac{\rho_{\text {water }} \cdot\left(\partial V_{\text {water }} / \partial t\right)}{M W_{\text {water }}}\right]
\end{gathered}
$$

Indeed, as peristaltic pumps were used to introduce fluids within the prototypes, $\mathrm{m}$ and $\mathrm{M}$ values have to be inferred from their respective volumetric quantities with Q_IPA $=0.785 \mathrm{~g} / \mathrm{cm}^{3}$ and Q_Ethanol $=0.789 \mathrm{~g} / \mathrm{cm}^{3}$. 


\subsection{Generic Design for Monitoring Excess Enthalpies}

Heat flow meters (HFMs) are nowadays quite common self generating sensors [2,3] allowing to measure heat transfers with low noise due to their reduced output impedance $(Z<100 \Omega)$. A generic structure, based on a planar thermopile, is depicted in Figure 1. Then, we investigated mixings with a first family of devices fabricated with PET 3D printing units relying on such sensors.

In any case an isothermal condition must be imposed on both liquids to be mixed. A mixing chamber (whose top is thermally insulated) was arranged on the upper face of a HFM whose other face was assembled on a heat sink.

Considering a planar thermopile structure involving $\mathrm{N}$ couples of thermojunctions, the amplified Seebeck output voltage representative of the heat current [Фmes $(W)]$ proceeding through the acting surface is:

$$
\mathrm{V}_{\text {out }}=\mathrm{G} \cdot \mathrm{Rs} \cdot \Phi=\mathrm{G} \cdot \mathrm{N} \cdot \Delta \alpha \cdot \Delta \theta
$$

where $G$ is the gain of the amplifier $(50<\mathrm{G}<1000)$, Rs the responsivity of the HFM (V/W), $\Delta \alpha$ the relative Seebeck coefficient $(\Delta \alpha \# 38 \mu \mathrm{V} / \mathrm{K})$ and $\delta \theta$ the local elementary differences in temperature available between two adjacent thermonctions as depicted in Figure 1a. Although quite liable, such systems based on HFMs featured a major drawback due to internal turbulence within the mixing chamber: then, a significant enhancement regarding the operating principle had to be considered.

\subsection{Specific Design with Micro Technology}

Any extraneous noise due to uncontrolled turbulence may be viewed as a significant drawback while considering the most common control processes where the flow of a solute has to be adjusted for a given flow of the solvent, the enthalpy of mixing being determined with prior assessments. To overcome such an issue, it is possible to locally ensure the mixing process upon one thermojunction out of two of the thermopile as depicted in Figure 2, the other one being at the reference temperature - that of both constituents-before mixing.

Then, we may now consider the thermopile output Seebeck e.m.f. given with the right term of Equation (4): it comes up as representative of a statistics on thermoelectric voltages localized on the numerous sets of thermojunctions: we verified that it could be considered as a valuable output signal practically associated with the mixing heat flow described with Equation (3b). Then, regarding a given mixing process in steady state with an expected theoretical heat flow rate $\Phi$ for a fixed water flow setpoint value, we may consider after amplification:

$$
\mathrm{V}_{\text {out }}=\mathrm{G} \cdot \xi \cdot \Phi
$$

with $\zeta(\mathrm{V} / \mathrm{W})$ an apparatus-related constant depending on the design of the thermopile and its overlaying channels driving both constituents.

\section{Results and Brief Discussion: Technological Achievement and Example of Output Signals}

As depicted in Figure 3 the above mentioned thermopile is arranged on a glass substrate and walls made of SU8 allow an apt repartition of both constituents to be mixed together.

Considering a controlled water flow fixed at $7.2 \pm 0.2 \mathrm{~mL} / \mathrm{min}$, we illustrate output signals volumetric flow values obtained with IPA and Ethanol in Figure $4 \mathrm{~b}$. With such a configuration the $\zeta$ parameter binding the thermopile emf and the theoretical flow is: $\zeta=2.083 .10^{-4} \mathrm{~V} / \mathrm{W}$. However, we must underline a slight $\zeta$-dependence on the water flow set point values. 


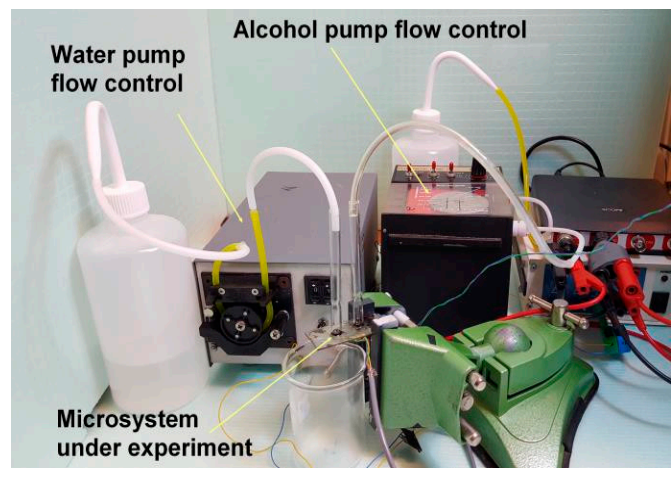

(a)

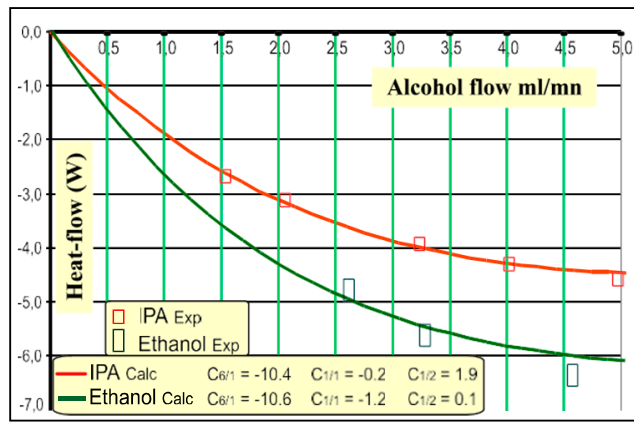

(b)

Figure 4. (a) Global view of the characterization system $(G=1000)$. (b) Compared values regarding measurements $\Phi_{\text {mes }}=V_{\text {out }} / G$. $\zeta$ and theoretical expected values calculated from Equation (3b).

\section{References}

1. Bejan, A. Entropy Generation Through Heat and Fluid Flow; Wiley \& Sons: Hoboken, NJ, USA, 1960.

2. Shirtliffe, C.J.; Tye, R.P. Guarded Hot Plate and Heat Flow Meter Methodology; ASTM Special Technical Publication: Philadelphia, PA, USA, 1985.

3. Gaviot, E.; Failleau, G.; Camberlein, L.; Polet, F.; Morice, R.; Bêche, B. Metrological prospects for the assessment of transition plateaus. Metrologia 2010, 47, 349-356, doi:10.1088/0026-1394/47/4/001.

4. Gaviot, E.; Failleau, G.; Camberlein, L.; Polet, F.; Morice, R.; Bêche, B. Towards a thermodynamic assessment of transition plateaus. Metrologia 2010, 47, 357-362, doi:10.1088/0026-1394/47/4/002.

5. Giordani, N.; Camberlein, L.; Gaviot, E.; Polet, F.; Bêche, B. Design and experimental validation of SU-8 based Micro-psychrometers. IEEE Trans. Instrum. Meas. Technol. 2007, 56, 102-106.

6. Boyne J.A.; Williamson A.G. Enthalpies of mixing of Ethanol and Water at $25{ }^{\circ}$ C. J. Chem. Eng. Data 1967, $12,318$.

7. Peeters D.; Huyskens P. Endothermicity or exothermicity of water/alcohol mixtures. J. Mol. Struct. 1993, 300, 539-550.

(C) 2018 by the authors. Licensee MDPI, Basel, Switzerland. This article is an open access article distributed under the terms and conditions of the Creative Commons Attribution (CC BY) license (http://creativecommons.org/licenses/by/4.0/). 